\title{
Bovine K-casein inhibits human rotavirus (HRV) infection via direct binding of glycans to HRV
}

\author{
M. Inagaki, ${ }^{\star}$ H. Muranishi,† K. Yamada,‡ K. Kakehi,‡ K. Uchida,§ T. Suzuki, ${ }^{\star}$ T. Yabe, ${ }^{\star} †$ T. Nakagomi,\# \\ O. Nakagomi,\# and Y. Kanamaru* ${ }^{1}$ \\ *United Graduate School of Agricultural Science, and \\ †Faculty of Applied Biological Sciences, Gifu University, 1-1 Yanagido, Gifu, Gifu 501-1193, Japan \\ ‡Faculty of Pharmaceutical Science, Kinki University, 3-4-1 Kowakae, Higashi-Osaka, Osaka 577-8502, Japan \\ §Central R\&D Laboratory, Kobayashi Pharmaceutical Co. Ltd., 1-30-3 Toyokawa, Ibaraki, Osaka 567-0057, Japan \\ \#Department of Molecular Microbiology and Immunology, Graduate School of Biomedical Sciences and Global Center of Excellence, \\ Nagasaki University, 1-12-4 Sakamoto, Nagasaki, Nagasaki 852-8523, Japan
}

\begin{abstract}
Human rotavirus (HRV) is a major etiologic agent of severe infantile gastroenteritis. $\kappa$-Casein $(\kappa-\mathrm{CN})$ from both human and bovine mature milk has been reported to have anti-HRV activity; however, the mechanism of this activity is poorly understood. The present study examined the molecular basis for the protective effect of bovine $\kappa$-CN derived from late colostrum (6-7 d after parturition) and from mature milk. Among the components of casein, $\mathrm{k}-\mathrm{CN}$ is the only glycosylated protein that has been identified. Therefore, we investigated whether the glycan residues in $\kappa-\mathrm{CN}$ were involved in the anti-HRV activity. Desialylated CN obtained by neuraminidase treatment exhibited anti-HRV activity, whereas deglycosylated $\mathrm{CN}$ obtained by o-glycosidase treatment lacked antiviral activity, indicating that glycans were responsible for the antiviral activity of CN. Furthermore, an evanescent-field fluorescence-assisted assay showed that HRV particles directly bound to heated casein (at $95^{\circ} \mathrm{C}$ for $30 \mathrm{~min}$ ) in a viral titer-dependent manner. Although the heated $\kappa$ - $\mathrm{CN}$ retained inhibitory activity in a neutralization assay, the activity was weaker than that observed before heat treatment. Our findings indicate that the inhibitory mechanism of bovine $\mathrm{k}-\mathrm{CN}$ against HRV involves direct binding to viral particles via glycan residues. In addition, heatlabile structures in $\mathrm{\kappa}$-CN may play an important role in maintenance of $\kappa-\mathrm{CN}$ binding to HRV.
\end{abstract}

Key words: human rotavirus, $\kappa$-casein, colostrum

\section{INTRODUCTION}

Rotavirus is an important cause of severe gastroenteritis in infants and young children worldwide. It is

Received December 3, 2013.

Accepted January 17, 2014.

${ }^{1}$ Corresponding author: kanamaru@gifu-u.ac.jp estimated that $95 \%$ of all children are infected with human rotavirus (HRV) by 5 yr of age, and such infections result in approximately $5 \times 10^{5}$ deaths annually (Parashar et al., 1998). Many studies on passive protection against HRV diarrhea have been conducted using either bovine colostrum or immunoglobulin concentrates prepared from bovine milk (Ebina et al., 1992; Sarker et al., 1998).

Colostrum contains high levels of bioactive agents and antibodies; however, bovine milk obtained within $5 \mathrm{~d}$ of parturition is not approved for sale in Japan. A previous study demonstrated the potential of using skimmed, sterilized, and concentrated bovine late colostrum (SCBLC) produced by healthy cows at 6 to $7 \mathrm{~d}$ after parturition as a prophylactic food additive against HRV gastroenteritis (Inagaki et al., 2012). Additionally, SCBLC has shown antiviral activity against norovirus (Murakami et al., 2010) and influenza virus (Uchida et al., 2012).

Previous studies have identified bovine mucin 1 (Kvistgaard et al., 2004), lactophorin (Inagaki et al., 2010, 2013), and PAS-6/7 (Inagaki et al., 2010, 2013) as nonimmunoglobulin components exhibiting inhibitory activity against HRV infection. In addition, $\kappa-\mathrm{CN}$ from both human and bovine mature milk has been reported to have anti-HRV effects in an international patent (Mukerji et al., 1996); however, its inhibitory mechanism against HRV remains to be elucidated.

Casein accounts for about $80 \%$ of proteins in bovine milk, and consists of 3 phosphoproteins, $\alpha_{\mathrm{S}^{-}}, \alpha_{\mathrm{S} 2^{-}}$, and $\beta-\mathrm{CN}$, and the phosphoglycoprotein $\kappa-\mathrm{CN}$, which is unique in having $3 \mathrm{O}$-glycosylation sites $\left(\mathrm{Thr}_{131}, \mathrm{Thr}_{133}\right.$, and $\mathrm{Thr}_{135}$; Eigel et al., 1984). Interestingly, the glycan structure of $\kappa-\mathrm{CN}$ has been reported to change during the transition from colostrum to mature milk (Saito et al., 1981). The current study focused on the involvement of glycans in the anti-HRV activity of CN, because glycans, including sialic acids such as $N$-acetylneuraminic acid (NeuAc), have been suggested to interact with HRV (Isa et al., 2006). 
The present study examined the anti-HRV activities of $\kappa$-CN derived from SCBLC and mature milk and demonstrated that direct binding of $\kappa-\mathrm{CN}$ to $\mathrm{HRV}$ via its glycan residues underlies its anti-HRV effects. This mechanism was elucidated by using both conventional viral neutralization assays and novel evanescent fieldbased fluorescence methods, which were previously used to investigate glycan-protein interactions (Kuno et al., 2005; Tateno et al., 2008). Furthermore, analyses using normal phase (NP)-HPLC and matrix-assisted laser desorption/ionization quadruple ion trap time-of-flight mass spectrometry (MALDI TOF-MS) revealed that the o-linked glycan compositions in $\mathrm{CN}$ differed between SCBLC and mature milk: monosialylated and disialylated oligosaccharides having a core 2 structure $\{N$-acetylglucosamine (GlcNAc) $\beta 1-6$ [galactose $(\mathbf{G a l})$ $\beta 1-3] \mathrm{N}$-acetylgalactosamine (GalNAc) $\}$ were detected in SCBLC but not in mature milk.

\section{MATERIALS AND METHODS}

\section{Materials, Cells, and Viruses}

All chemicals used in these studies were of reagent grade. Cells of the Rhesus monkey kidney cell line MA104 were cultured in Eagle's Minimal Essential Medium (MEM) supplemented with $10 \%$ fetal bovine serum (FBS). Human rotavirus MO strain (serotype G3P [8]) was propagated and the virus titer was determined by assaying fluorescent cell focus-forming units (FCFU), as previously described (Ebina et al., 1990). Human rotavirus treated with trypsin (Type IX-S, Sigma-Aldrich, St. Louis, MO) at $37^{\circ} \mathrm{C}$ for 30 min was used in infection assays and glycan arrays that involved measurements of evanescent field-based fluorescence.

\section{Bovine Milk Samples}

Bovine mature milk was collected from healthy Holstein-Friesian cows held at Gifu University Farm (Japan). Large-scale batches of SCBLC from healthy cows were prepared at Kobayashi Pharmaceuticals (Osaka, Japan; Inagaki et al., 2012). Colostrum whey from a cow hyperimmunized with HRV (rota whey) was prepared as previously described (Ebina et al., 1992). Pure bovine $\kappa-\mathrm{CN}$ and bovine lactoferrin were purchased from Sigma-Aldrich and used as control samples. Bovine serum albumin fraction $\mathrm{V}$ was purchased from Roche Applied Science (Mannheim, Germany).

\section{Separation of $k-C N$ from Bovine Milk Samples}

Skim milk was prepared by centrifugation of bovine mature milk at $1,500 \times g$ for $20 \mathrm{~min}$ at $4^{\circ} \mathrm{C}$ to remove fat. Crude $\mathrm{CN}$ was prepared by isoelectric precipitation of skim milk at $\mathrm{pH}$ 4.6. The SCBLC (5 g) was dissolved in purified water $(1 \mathrm{~L})$ and precipitated at $\mathrm{pH} 4.6$ as crude $\mathrm{CN}$. The IgG fraction of crude $\mathrm{CN}$ was purified by Hitrap Protein G HP ( $5 \mathrm{~mL}$, GE Healthcare, Chalfont St. Giles, UK; Inagaki et al., 2013), and the unbound fraction was collected as CN.

Isolation of $\kappa$-CN from $\mathrm{CN}$ was carried out using anion exchange chromatography with a MonoQ 5/50 GL column (1 mL, GE Healthcare) as described by Plank et al. (2008) with slight modifications. Briefly, CN was dissolved in $20 \mathrm{~m} M$ imidazole-3.3 $M$ urea buffer containing $80 \mathrm{mM} \mathrm{NaCl}(\mathrm{pH} \mathrm{8.0)}$ at a concentration of 10 $\mathrm{mg} / \mathrm{mL}$. This solution was incubated with $0.2 \%$ (vol/ vol) 2-mercaptoethanol for $12 \mathrm{~h}$ at $4^{\circ} \mathrm{C}$ for reduction of disulfide bonds, and then applied to a column equilibrated with the same buffer at a flow rate of $1 \mathrm{~mL} /$ min. The bound fraction was eluted from the column with same buffer containing $0.8 \mathrm{M} \mathrm{NaCl}$. The 4 bound fractions [fractions (F)1 to 4] were collected according to the gradient pattern of elution buffer (elution program described in legend for Supplemental Figure S1; http://dx.doi.org/10.3168/jds.2013-7792), and each fraction was dialyzed against distilled water and then lyophilized. Fractions were separated by SDS-PAGE using the Laemmli (1970) method, and the gels were stained with Coomassie Brilliant Blue.

\section{Isolation of Glycopeptides from Trypsinized CN}

The glycopeptides produced by trypsinization of CN were isolated using Arachis hypogaea peanut agglutinin (PNA) lectin affinity chromatography on a PNAHPLC column (2.5 mL, J-Oil Mills Inc., Tokyo, Japan) using the following procedure. The $\mathrm{CN}$ was incubated for $24 \mathrm{~h}$ at $37^{\circ} \mathrm{C}$ with L-1-tosylamide-2-phenylethyl chloromethyl ketone (TPCK)-treated trypsin (SigmaAldrich) in $50 \mathrm{mM}$ Tris-HCl buffer (pH 8.0) at an enzyme:substrate ratio of 1:50 (wt/wt). The digestion process was terminated by boiling, and the tryptic digest of $\mathrm{CN}$ was diluted $\sim 10$-fold with $50 \mathrm{~m} M$ Tris$\mathrm{H}_{2} \mathrm{SO}_{4}$ buffer ( $\mathrm{pH}$ 7.3). This solution was then loaded onto a PNA-HPLC column equilibrated with the same buffer at a flow rate of $0.4 \mathrm{~mL} / \mathrm{min}$. The bound fraction was eluted and later collected with the same buffer containing $50 \mathrm{mM} \mathrm{D}(+)$-galactose.

\section{Desialylation and Deglycosylation Treatments of CN}

Casein was dissolved in $50 \mathrm{~m} M$ ammonium acetate ( $\mathrm{pH}$ 5.5). Neuraminidase from Vibrio cholerae (Roche Applied Science) was added to the sample solution at $0.2 \mathrm{U} / \mathrm{mg}$ of substrate, and the mixture was incubated at $37^{\circ} \mathrm{C}$ for $26 \mathrm{~h}$. The reaction mixture was then boiled 
and dialyzed against distilled water, and the desialylated $\mathrm{CN}$ product was collected. Lectin binding activity after desialylation with neuraminidase was examined by dot blotting. The desialylated $\mathrm{CN}$ was immobilized onto a polyvinylidene difluoride membrane (PVDF; Millipore, Bedford, MA) using a vacuum dot blotter (Sanplatec, Osaka, Japan). Blotted peptides were detected using biotinylated Triticum vulgaris wheat germ agglutinin (WGA) lectin (J-Oil Mills Inc.) and horseradish peroxidase-labeled streptavidin, and visualized with 3,3'-diaminobenzidine.

o-Glycosidase from Streptococcus pneumoniae (Roche), which releases the Gal $\beta 1-3$ GalNAc ( $\mathrm{T}$ antigen: core 1) unit from $O$-glycoproteins was added to desialylated $\mathrm{CN}$ dissolved in $50 \mathrm{~m} M$ Tris- $\mathrm{HCl}$ buffer (pH 6.5) at $2 \mathrm{mU} / \mathrm{mg}$ of substrate, and the mixture was incubated for $24 \mathrm{~h}$ at $37^{\circ} \mathrm{C}$. The reaction mixture was then boiled and dialyzed against distilled water, and the deglycosylated $\mathrm{CN}$ was collected. The lectin binding activity remaining after deglycosylation with $O$ glycosidase was immobilized and detected by the same method using Agaricus bisporus agglutinin (ABA) lectins (J-Oil Mills Inc.).

\section{Rotavirus Infection Assays}

The replication inhibition (neutralization) assay for HRV MO strain was conducted using MA104 cells as previously described (Inagaki et al., 2013). Briefly, MA104 cells were seeded onto 24-well heavy Tefloncoated slides (AR Brown, Tokyo, Japan) and grown to full confluence. Trypsinized HRV was diluted with MEM containing $2 \%$ FBS at a titer of $\sim 1 \times 10^{3}$ FCFU per $100 \mu \mathrm{L}$, and was mixed with equal volumes (100 $\mu \mathrm{L}$ ) of samples that had been serially diluted with MEM containing $2 \% \mathrm{FBS}$ for $1 \mathrm{~h}$ at $37^{\circ} \mathrm{C}$. Then, the diluted mixtures were inoculated to the confluent monolayers of MA104 cells. Control samples without the test sample of milk showed approximately 100 infected foci per well. The cells were cultured for $1 \mathrm{~h}$ at $37^{\circ} \mathrm{C}$ in $5 \% \mathrm{CO}_{2}$. After removal of the inocula, the cells were washed with MEM to remove unbound virus and then incubated for $17 \mathrm{~h}$ at $37^{\circ} \mathrm{C}$ in $5 \% \mathrm{CO}_{2}$. Following incubation, the cells were fixed by addition of cold methanol, and infected cells were detected by indirect immunofluorescence assay using a monoclonal antibody against virus protein 6 (Minamoto et al., 1993) and fluorescein isothiocyanate-conjugated anti-mouse $\operatorname{IgG}$ goat serum (American Qualex, San Clemente, CA); the foci were counted under a fluorescence microscope. Inhibitory activity was expressed as the percentage reduction in foci number of infected cells compared with infected cells without the milk sample. The MIC (the minimum concentration showing a $50 \%$ reduction of infected cells) for each sample was calculated using a logistic regression of the concentration-dependent percentage of foci reduction.

Pretreatment assays previously described by Kvistgaard et al. (2004) were conducted with slight modifications to investigate for interactions of milk sample with cells as antiviral activity. Serially diluted milk samples were inoculated to the confluent monolayers of MA104 cells by incubation for $1 \mathrm{~h}$ at $37^{\circ} \mathrm{C}$ in $5 \% \mathrm{CO}_{2}$. Following incubation, the cells were washed once with the same medium, and the trypsinized HRV, diluted with MEM containing $2 \%$ FBS at a titer of $\sim 2 \times 10^{3}$ FCFU per $100 \mu \mathrm{L}$, was inoculated to cells. Controls without milk test samples showed $\sim 100$ infected foci per well. After removal of the viral inoculation, the cells were washed and incubated for $17 \mathrm{~h}$ at $37^{\circ} \mathrm{C}$ in $5 \%$ $\mathrm{CO}_{2}$. Subsequent fixation, detection of infected cells, and MIC determinations were performed as described above.

Concentrations of milk protein and glycopeptides were determined using a Bradford Protein Assay Kit (Bio-Rad, Hercules, CA) and a BCA Protein Assay Kit (Thermo Fisher Scientific, Waltham, MA), and BSA was used as the protein standard.

\section{Analysis of O-Linked Glycans in CN}

The o-linked glycans in CN were analyzed as previously described (Yamada et al., 2007). Briefly, an aqueous solution of purified $\mathrm{CN}(200 \mu \mathrm{g} / 80 \mu \mathrm{L})$ was injected into an automated glycan releasing system (AutoGlycoCutter, Shimadzu, Kyoto, Japan; Yamada et al., 2007, 2010), which released the reducing $o$-linked glycans from the core protein structure. The released $o$-linked glycans were evaporated to dryness with a centrifugal evaporator, dissolved in 2-aminobenzoic acid solution, and stored at $80^{\circ} \mathrm{C}$ for $1 \mathrm{~h}$. After cooling, water $(100 \mu \mathrm{L})$ was added to the dried residue and the solution was applied to a Sephadex LH-20 (GE Healthcare) gel filtration column equilibrated with $50 \%$ aqueous methanol. The eluted fluorescent fractions were pooled and evaporated to dryness under reduced pressure. The dried residues were dissolved in water (50 $\mu \mathrm{L}$ ), and $20 \mathrm{~mL}$ aliquots were analyzed by NP-HPLC. The HPLC separations were done using a TSK-GEL Amide-80 column (Tosoh, Tokyo, Japan) and a linear gradient consisting of $0.1 \%$ acetic acid in acetonitrile (solvent $\mathrm{A}$ ) and $0.2 \%$ acetic acid in water containing $0.1 \%$ triethylamine (solvent B) at $40^{\circ} \mathrm{C}$. The column was initially equilibrated and eluted with $15 \%$ solvent $\mathrm{B}$ for $2 \mathrm{~min}$; then, solvent B was increased to $50 \%$ over $80 \mathrm{~min}$ at $1.0 \mathrm{~mL} / \mathrm{min}$. 


\section{Detection of HRV-CN Interactions by Evanescent Field-Based Fluorescence-Assisted Detection}

Samples were dissolved in 50\% (vol/vol) spotting buffer (Rexxam, Osaka, Japan), and $1 \mu \mathrm{L}$ of each sample $(0.5 \mathrm{mg} / \mathrm{mL})$ was spotted into an epoxy-coated glass well. The spotted samples were then incubated for 12 $\mathrm{h}$ at room temperature in a moist chamber. Following incubation, excess nonimmobilized materials were washed out, and unreacted epoxy groups were blocked with blocking buffer (Rexxam) at room temperature for $1.5 \mathrm{~h}$. Then, trypsinized HRV was diluted with binding buffer (Rexxam) at titers of $6.2 \times 10^{4}$ to $3.8 \times 10^{3}$ FCFU per $80 \mu \mathrm{L}$, and an equal volume $(80 \mu \mathrm{L})$ was added to each well, followed by incubation for $1 \mathrm{~h}$ with shaking. Following removal of excess HRV, the IgG purified from rota whey and cyanin 3-labeled rabbit anti-bovine IgG (Jackson Immunoresearch Laboratories Inc., West Grove, PA) were added to the well and the fluorescent intensity within several hundred nanometers from the well surface was measured by evanescent field-based fluorescence using a Bio-Rex scan 200 fluorescence scanner (Rexxam).

\section{RESULTS AND DISCUSSION}

\section{Inhibitory Activity of $\mathrm{k}-\mathrm{CN}$ Against HRV Infection}

The inhibitory activity of CN derived from SCBLC and mature milk against HRV infection was determined using the neutralization assay. Both derived caseins demonstrated $50 \%$ inhibitory activity at a sample concentration of $0.1 \mu \mathrm{g} / \mathrm{mL}$; however, SCBLC CN showed stronger inhibitory potency than did $\mathrm{CN}$ from mature milk (Figure 1A).

To confirm the anti-HRV activity of $\kappa-\mathrm{CN}$, we attempted to separate $\kappa-\mathrm{CN}$ from both SCBLC and mature milk by anion exchange chromatography, as shown in Supplemental Figure S1 (http://dx.doi.org/10.3168/ jds.2013-7792). Five fractions (an unbound fraction and 4 bound fractions, F1, F2, F3, and F4) were collected according to the elution pattern. The main elution peak containing $\kappa$-CN was $\mathrm{F} 2$. This was confirmed by results of SDS-PAGE analysis, which showed a band with a molecular size of $28 \mathrm{kDa}$ corresponding to pure $\kappa-\mathrm{CN}$ as the main component for both SCBLC and mature milk (Supplemental Figure S2; http://dx.doi.org/10.3168/ jds.2013-7792).

The inhibitory effect of F2 against HRV infection was examined by a neutralization assay. The MIC values of F2 separated from SCBLC and mature milk were 0.021 and $0.12 \mu \mathrm{g} / \mathrm{mL}$, respectively. These inhibitory activities were much greater than that of bovine lactoferrin, which showed a MIC of $180 \mu \mathrm{g} / \mathrm{mL}$ (Figure 1B). Frac- tion 2 from both SCBLC and mature milk displayed concentration-dependent anti-HRV activity in the range of 0.001 to $0.1 \mu \mathrm{g} / \mathrm{mL}$, and activity reached a plateau of $\sim 70 \%$ inhibition at a concentration of $\sim 1 \mu \mathrm{g} / \mathrm{mL}$. Fraction 2 from SCBLC was stronger than F2 from mature milk in inhibiting infection of cells by HRV. In contrast to F2, F1, F3, and F4 were not detected to have anti-HRV activity, even at concentrations of 100 $\mu \mathrm{g} / \mathrm{mL}$ (data not shown).


Figure 1. Effects of $\kappa$-CN fractions on human rotavirus (HRV) infection. (A) Inhibitory activity of CN derived from skimmed, sterilized, and concentrated bovine late colostrum (SCBLC; $\bullet$ ) and mature milk $(\bigcirc)$ was measured by a neutralization assay as described in Materials and Methods. Control cells were assayed only for virus without sample. Inhibitory activity of various samples is expressed as the percentage decrease in infected cells compared with the level of control cells (adjusted to 100\%). Results are expressed as means \pm standard deviations (SD) of 3 independent experiments conducted in triplicate. (B) Inhibitory activity of $\kappa$-CN containing fractions by neutralization assay. The samples used were fraction 2 (F2) from CN of SCBLC $(\bullet)$ and mature milk $(\bigcirc)$, and bovine lactoferrin $(\Delta)$. Results are expressed as means \pm SD of 3 independent experiments, conducted in triplicate. 
A


B


Figure 2. Effects of desialylated $\mathrm{CN}$ and deglycosylated $\mathrm{CN}$ on human rotavirus (HRV) infection. (A) Lectin binding reactivity of CN before and after enzymatic digestion. Samples of CN treated or not treated with neuraminidase were immobilized by dot blotting and detected by wheat germ agglutinin (WGA) lectin. Samples of desialylated $\mathrm{CN}$ with and without $O$-glycosidase treatment were immobilized by dot blotting and detected by Agaricus bisporus agglutinin (ABA) lectin. Inhibitory activity of $\mathrm{CN}$ derived from skimmed, sterilized, and concentrated bovine late colostrum (SCBLC; panel B) and mature milk (panel C) with enzymatic modification was measured by the neutralization assay. The samples used were SCBLC CN (•); SCBLC CN treated with neuraminidase (desialylated SCBLC; $\mathbf{\square})$; SCBLC CN treated with o-glycosidase (deglycosylated SCBLC; $\mathbf{\Delta}$ ); mature milk CN (O); mature milk $\mathrm{CN}$ treated with neuraminidase (desialylated mature milk; $\square$ ); mature milk CN treated with o-glycosidase (deglycosylated mature milk; $\Delta$ ). Results are expressed as means \pm standard deviation of 3 independent experiments conducted in triplicate.
Deglycosylated CN Did Not Exhibit Anti-HRV Activity

$\kappa$-Casein is the only glycosylated protein among the CN components (Eigel et al., 1984). To investigate the involvement of $\kappa$-CN glycans in anti-HRV activity, $\mathrm{CN}$ was subjected to deglycosylation with neuraminidase and o-glycosidase. As shown in Figure 2A, CN derived from SCBLC and mature milk did not exhibit binding to WGA after being treated with neuraminidase, indicating cleavage of NeuAc. However, desialylated CN retained its potent inhibitory activity against HRV, with a MIC $<1.0 \mu \mathrm{g} / \mathrm{mL}$, which was almost identical to the activity of $\mathrm{CN}$ without neuraminidase treatment (Figures 2B and $2 \mathrm{C}$ ). These results suggest that NeuAc residues are not involved in the HRV inhibitory activity of CN.

Next, samples of CN were treated with o-glycosidase. This enzyme releases the core 1 unit, the major constituent glycan unit in bovine $\kappa-\mathrm{CN}$ (Eigel et al., 1984). The samples treated with $o$-glycosidase were analyzed for their ability to bind ABA, which is known to detect core 1-related o-linked glycans (Nakamura-Tsuruta et al., 2006). Following o-glycosidase treatment, deglycosylated CN from SCBLC clearly showed diminished ABA binding capacity compared with untreated $\mathrm{CN}$. Additionally, the reactivity of deglycosylated CN from mature milk treated with $o$-glycosidase was eliminated after treatment (Figure 2A). Remarkably, deglycosylated caseins from SCBLC and mature milk failed to exhibit any inhibitory activity against HRV (Figures $2 \mathrm{~B}$ and $2 \mathrm{C}$ ), indicating that $o$-linked glycans in $\mathrm{CN}$ are essential for inhibitory activity against HRV infection.

To better understand the glycan motifs involved in anti-HRV activity, reducing oligosaccharides were released from CN from SCBLC and mature milk using the AutoGlycoCutter. The released oligosaccharide residues were then labeled with 2-aminobenzoic acid and analyzed by NP-HPLC and MALDI-TOF MS. Striking differences were observed between the oligosaccharides released from SCBLC and mature milk (Figure 3). Disialyl T antigen [NeuAc $\alpha 2-3$ Gal $\beta 1-3$ (NeuAc $\alpha 2-6$ ) GalNAc] was predominant in mature milk (Figure 3, peak 4), whereas sialylated and disialylated antigens having core 2 structures were detected in SCBLC but not in mature milk (Figure 3, peaks 5 and 6). These antigens are known to be present at the early stage (within $3 \mathrm{~d}$ after giving birth) of lactation but are downregulated in later stages (Saito and Itoh, 1992; Wilson et al., 2008).

\section{Inhibitory Activity of CN Glycopeptides Is Attributed to Interaction with HRV}

Our studies demonstrated that glycans in $\mathrm{CN}$ are the primary structures involved in HRV inhibitory activity, 


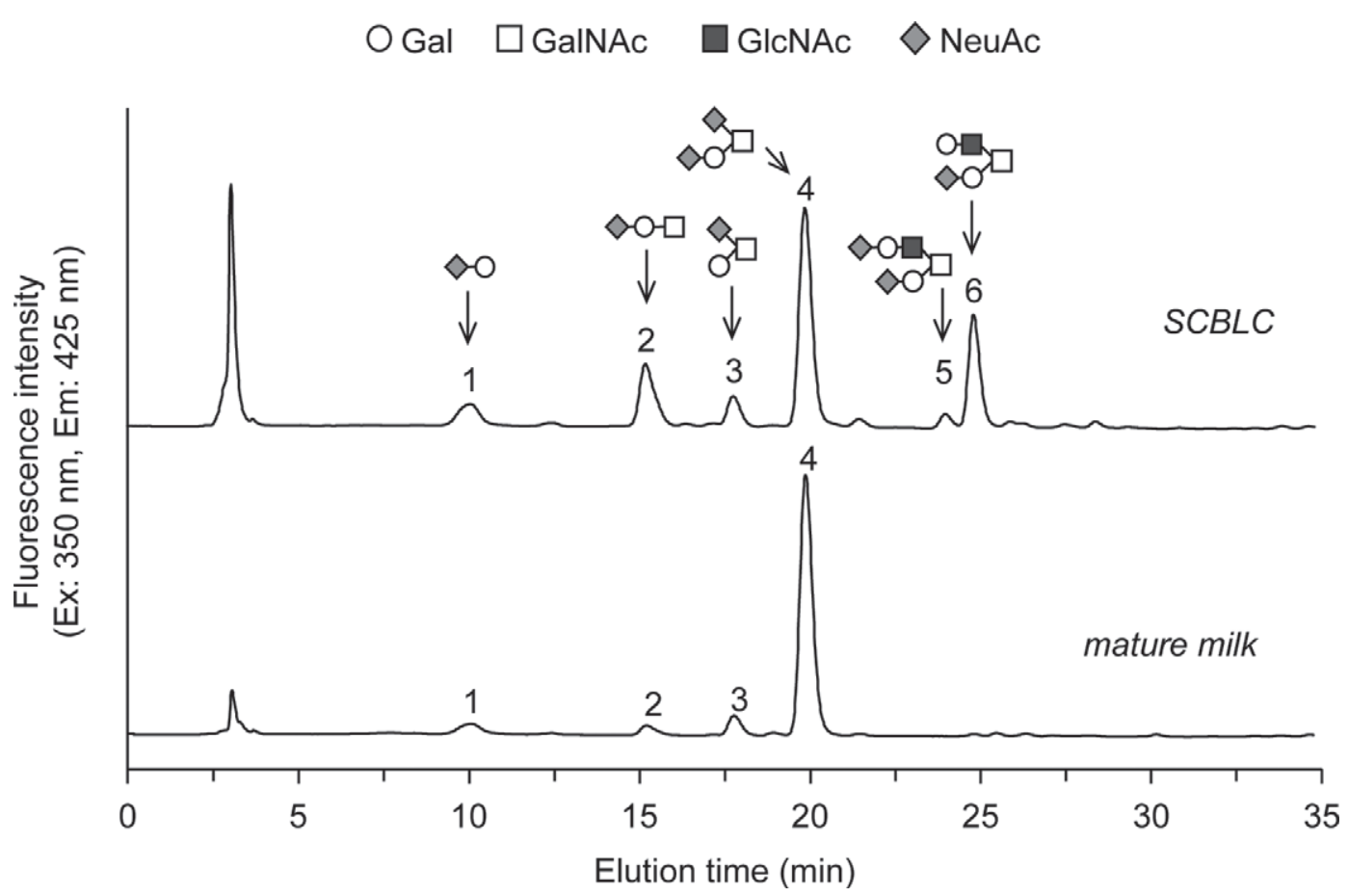

Figure 3. Normal phase-HPLC analysis of o-linked glycans derived from CN of skimmed, sterilized, and concentrated bovine late colostrum (SCBLC) and mature milk using TSK-GEL Amide-80 (Tosoh, Tokyo, Japan). See Materials and Methods for analytical conditions. Gal = galactose; GalNAc $=N$-acetylgalactosamine; GlcNAc $=N$-acetylglucosamine; NeuAc $=N$-acetylneuraminic acid.

and we therefore isolated the glycopeptides from trypsinized CN, using PNA lectin affinity chromatography to isolate core 1. The PNA-bound glycopeptides were examined in a neutralization assay. Glycopeptides from both SCBLC and mature milk exhibited anti-HRV activity in the neutralization assay (both MIC were $<0.01 \mu \mathrm{g} / \mathrm{mL}$, Figure $4 \mathrm{~A}$ ), indicating that glycans in $\mathrm{CN}$ were the molecular structures that conveyed inhibitory potency against HRV infection. The glycopeptides from SCBLC were more robust than those of mature milk in inhibiting HRV infection of host cells. We next conducted a pretreatment assay to investigate whether the inhibitory effect of glycopeptides against HRV resulted from its interaction with host cells. The results clearly demonstrated that interaction of glycopeptides with host cells did not affect anti-HRV activity (Figure $4 \mathrm{~B}$ ) and suggest that glycans in $\mathrm{CN}$ interact with viral particles, but not with host cells.

\section{Heat-Treated CN Directly Interacts with HRV Particles}

To verify direct interaction of glycans in $\mathrm{CN}$ with HRV, we attempted to demonstrate HRV binding to $\mathrm{CN}$ after incubation for $30 \mathrm{~min}$ at $95^{\circ} \mathrm{C}$. Binding was detected by evanescent field-based fluorescence detection, which is an established method for detection of weak interactions between glycans and various biological samples (or carbohydrate binding proteins; Kuno et al., 2005; Tateno et al., 2008). Spots for the heated CN from both SCBLC and mature milk were explicitly observed at a viral titer of $7.7 \times 10^{5} \mathrm{FCFU} / \mathrm{mL}$, but visible spots were not detected for BSA or bovine lactoferrin (Figure 5A). Unexpectedly, the binding of heated $\mathrm{CN}$ from mature milk was $\sim 1.5$-fold stronger than the binding of heated $\mathrm{CN}$ from SCBLC (Figure 5B). On the other hand, SCBLC-derived components appeared to have slightly higher anti-HRV activity than mature milk-derived components in a neutralization assay conducted using cultured cells (Figures 1A, 1B, 2B, 2C, and $4 \mathrm{~A}$ ). These findings suggest that binding intensity for HRV does not necessarily correlate with potency of inhibitory activity.

\section{Heat-Labile Structure in $\mathrm{k}-\mathrm{CN}$ Likely Participates in Anti-HRV Activity}

Finally, to confirm that the anti-HRV activity of $\kappa-\mathrm{CN}$ can be attributed to the structures of its glycans, we investigated the activity of F2 (major fraction of $\kappa-\mathrm{CN})$ as shown in Supplemental Figure S2 (http:// dx.doi.org/10.3168/jds.2013-7792), after incubation for $30 \mathrm{~min}$ at $95^{\circ} \mathrm{C}$. Surprisingly, the heated F2 from both SCBLC and mature milk demonstrated $\sim 50 \%$ 
A

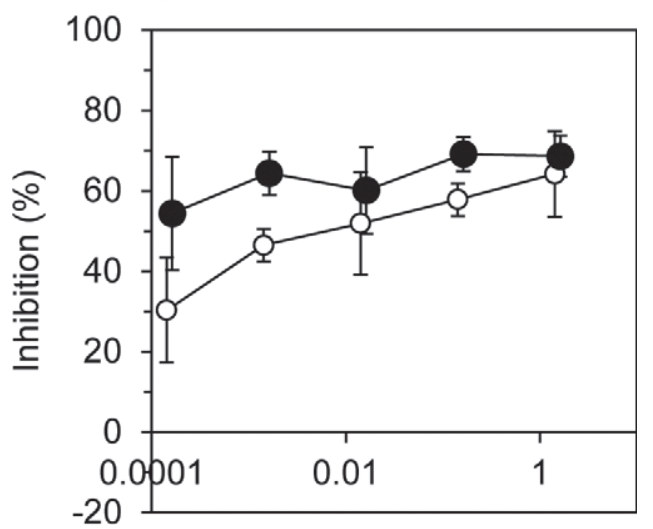

B

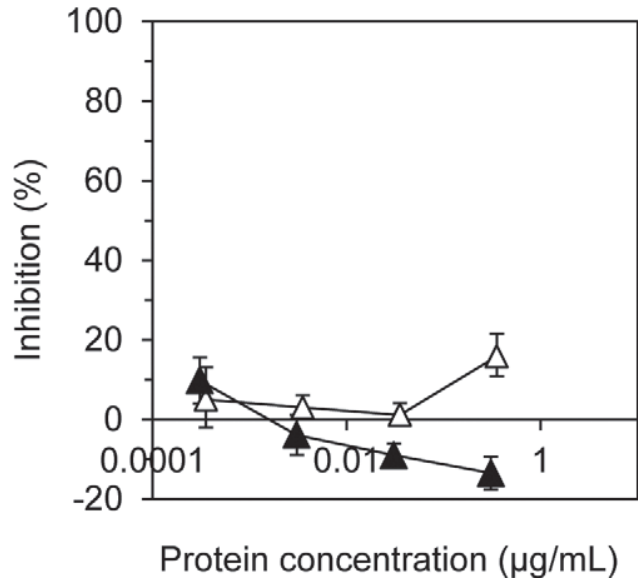

Figure 4. Inhibitory mechanism of $\mathrm{CN}$-derived glycopeptides that affect human rotavirus (HRV) infection of MA104 cells. (A) Inhibitory activity was determined by the neutralization assay. Samples evaluated in the neutralization assay were Arachis hypogaea peanut agglutinin lectin affinity (PNA-LA)-column bound fractions derived from $\mathrm{CN}$ of skimmed, sterilized, and concentrated bovine late colostrum (SCBLC; -) and mature milk $(\mathrm{O})$. (B) Inhibitory activity was determined by pretreatment assay as described in Materials and Methods. Control cells without samples were assayed only for virus. Inhibitory activity of various samples is expressed as the percentage decrease in infected cells compared with the level of the control cells (adjusted to 100\%). Samples evaluated in pretreatment assay were PNA-LA column-bound fractions derived from $\operatorname{SCBLC}(\boldsymbol{\Delta})$ and mature milk $(\Delta)$.

inhibitory activity at sample concentrations of $10 \mu \mathrm{g} /$ $\mathrm{mL}$ (Figure 6), although the inhibitory activities were weaker than activities observed before heat treatment (Figure 1B). Results shown in Figures 2B and 2C clearly show that $o$-linked glycans in $\mathrm{CN}$ are the key molecular structures involved in inhibitory activity against HRV infection; however, the reduction in anti-HRV activity shown by heated $\kappa-\mathrm{CN}$ suggests involvement of a heatlabile structural portion of $\mathrm{k}-\mathrm{CN}$, which might assist in recognition of HRV.
A

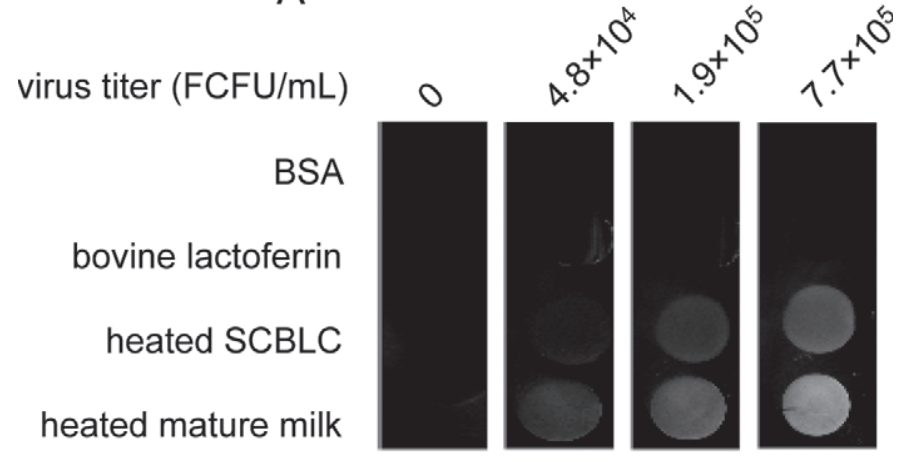

B



Virus titer (FCFU/mL)

Figure 5. Observation of human rotavirus (HRV)-milk protein interaction. (A) Photograph of HRV-milk protein interaction by evanescent field-based fluorescence detection. The samples used were BSA, bovine lactoferrin, and CN from both skimmed, sterilized, and concentrated bovine late colostrum (SCBLC) and mature milk heated for 30 min at $95^{\circ} \mathrm{C}$. (B) Fluorescence intensity of HRV-heated CN interaction by evanescent field-based fluorescence. The samples indicated are BSA $(\square)$, bovine lactoferrin $(\Delta)$, heated $\operatorname{SCBLC~CN~}(\bullet)$, and heated mature milk $\mathrm{CN}(\bigcirc)$. Results are expressed as means \pm standard deviations of 3 independent experiments conducted in triplicate. FCFU = fluorescent cell focus-forming units.

Several observations showed that the CN-derived test samples exhibited a plateau at 50 to $70 \%$ inhibitory activity against HRV infection in MA104 cells, and inhibition never reached $100 \%$. Although the reason for these results is unclear, both detectable but low binding affinity to virus particles and involvement of a heat-labile structural portion of the glycans in the inhibitory activity might partly explain these findings. Rotavirus infection is known to involve a multistep cell entry process, which seems to require sequential interaction of virus surface proteins with distinct cellsurface molecules in an organized membrane microenvironment, such as rafts (Baker and Prasad, 2010). The observation that glycans in $\kappa$-CN could not perfectly 


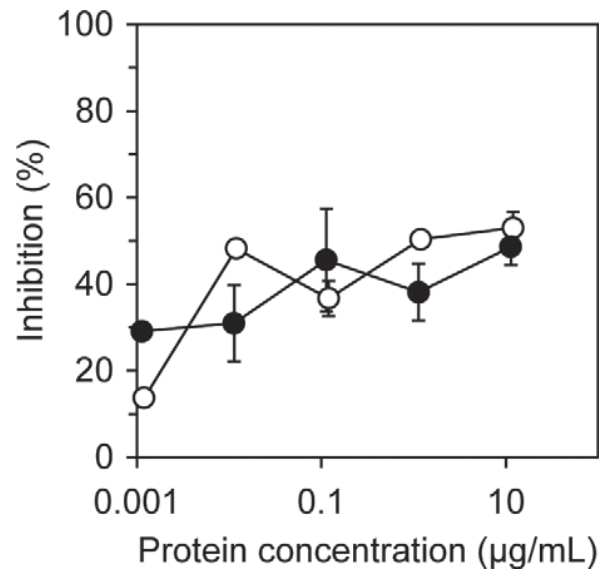

Figure 6. Effects of heated $\kappa$-CN containing fraction on human rotavirus (HRV) infection: inhibitory activities of fraction 2 (F2) of $\mathrm{k}$-CN after incubation at $95^{\circ} \mathrm{C}$ for $30 \mathrm{~min}$, as determined using the neutralization assay. The samples tested were heated F2 of skimmed, sterilized, and concentrated bovine late colostrum (SCBLC; $\bullet$ ) and mature milk $(\bigcirc)$. Results are expressed as means \pm standard deviations of 3 independent experiments conducted in triplicate.

inhibit HRV infection may imply the existence of one or several key molecules that play a significant role in the cell entry process.

\section{ACKNOWLEDGMENTS}

We thank Mayumi Yamamoto (Faculty of Applied Biological Sciences, Gifu University, Gifu, Japan) for her helpful technical assistance and Norihito Shimizu (Faculty of Applied Biological Sciences, Gifu University) for discussing this study with the investigators. We thank Nobuyuki Minamoto and Makoto Sugiyama (both of Faculty of Applied Biological Sciences, Gifu University) for kindly providing the monoclonal antibody for detection of HRV-infected cells. This research was supported by the Japan Society for the Promotion of Science [JSPS; Grant-in-Aid for Scientific Research (C) no. 21580325 (to Y. K.), Grant-in-Aid for Young Scientists (B) no. 24790044 (to K. Y.)].

\section{REFERENCES}

Baker, M., and B. V. Prasad. 2010. Rotavirus cell entry. Curr. Top. Microbiol. Immunol. 343:121-148.

Ebina, T., M. Ohta, Y. Kanamaru, Y. Yamamoto-Osumi, and K. Baba. 1992. Passive immunizations of suckling mice and infants with bovine colostrum containing antibodies to human rotavirus. J. Med. Virol. 38:117-123.

Ebina, T., K. Tsukada, K. Umezu, M. Nose, K. Tsuda, H. Hatta, M. Kim, and T. Yamamoto. 1990. Gastroenteritis in suckling mice caused by human rotavirus can be prevented with egg yolk immunoglobulin (IgY) and treated with a protein-bound polysaccharide preparation (PSK). Microbiol. Immunol. 34:617-629.

Eigel, W. N., J. E. Butler, C. A. Ernstrom, H. M. Farrell, V. R. Harwalkar, R. Jenness, and R. M. Whitney. 1984. Nomenclature of proteins of cow's milk: Fifth revision. J. Dairy Sci. 67:1599-1631.
Inagaki, M., S. Nagai, T. Yabe, S. Nagaoka, N. Minamoto, T. Takahashi, T. Matsuda, O. Nakagomi, T. Nakagomi, T. Ebina, and Y. Kanamaru. 2010. The bovine lactophorin C-terminal fragment and PAS6/7 were both potent in the inhibition of human rotavirus replication in cultured epithelial cells and the prevention of experimental gastroenteritis. Biosci. Biotechnol. Biochem. 74:1386-1390

Inagaki, M., Xijier, Y. Nakamura, T. Takahashi, T. Yabe, T. Nakagomi, O. Nakagomi and Y. Kanamaru. 2012. Production and functional properties of dairy products containing lactophorin and lactadherin. Pages 49-64 in Food Additives. Y. El-Samragy, ed. InTech, Rijeka, Croatia.

Inagaki, M., M. Yamamoto, Cairangzhuoma, Xijier, T. Yabe, K. Uchida, M. Kawasaki, T. Nakagomi, O. Nakagomi, and Y. Kanamaru. 2013. Multiple dose therapy with bovine colostrum confers significant protection against diarrhea in a mouse model of human rotavirus-induced gastrointestinal disease. J. Dairy Sci. 96:806-814.

Isa, P., C. F. Arias, and S. López. 2006. Role of sialic acids in rotavirus infection. Glycoconj. J. 23:27-37.

Kuno, A., N. Uchiyama, S. Koseki-Kuno, Y. Ebe, S. Takashima, M. Yamada, and J. Hirabayashi. 2005. Evanescent-field fluorescenceassisted lectin microarray: A new strategy for glycan profiling. Nat. Methods 2:851-856.

Kvistgaard, A. S., L. T. Pallesen, C. F. Arias, S. López, T. E. Petersen, C. W. Heegaard, and J. T. Rasmussen. 2004. Inhibitory effects of human and bovine milk constituents on rotavirus infections. J. Dairy Sci. 87:4088-4096.

Laemmli, U. K. 1970. Cleavage of structural proteins during the assembly of the head of bacteriophage T4 . Nature 227:680-685.

Minamoto, N., O. Sugimoto, M. Yokota, M. Tomita, H. Goto, M. Sugiyama, and T. Kinjo. 1993. Antigenic analysis of avian rotavirus VP6 using monoclonal antibodies. Arch. Virol. 131:293-305.

Mukerji, P., P. A. Preito, A. E. Seo, J. H. Baxter, and R. D. Cummings, inventors. 1996. Inhibition of human rotavirus infection. Abbott Laboratories, assignee. US Pat. No. WO9608269.

Murakami, K., S. Suzuki, N. Aoki, T. Okajima, D. Nadano, K. Uchida, K. Yamashita, T. Oka, K. Katayama, N. Takeda, and T. Matsuda. 2010. Binding of norovirus virus-like particles (VLPs) to human intestinal Caco-2 cells and the suppressive effect of pasteurized bovine colostrum on this VLP binding. Biosci. Biotechnol. Biochem. 74:541-547.

Nakamura-Tsuruta, S., J. Kominami, A. Kuno, and J. Hirabayashi 2006. Evidence that Agaricus bisporus agglutinin (ABA) has dual sugar-binding specificity. Biochem. Biophys. Res. Commun. $347: 215-220$.

Parashar, U. D., J. S. Bresee, J. R. Gentsch, and R. I. Glass. 1998. Rotavirus. Emerg. Infect. Dis. 4:561-570.

Plank, J., P. R. Andres, I. Krause, and C. Winter. 2008. Gram scale separation of casein proteins from whole casein on a Source $30 \mathrm{Q}$ anion-exchange resin column utilizing fast protein liquid chromatography (FPLC). Protein Expr. Purif. 60:176-181.

Saito, T., T. Itoh, S. Adachi, T. Suzuki, and T. Usui. 1981. The chemical structure of neutral and acidic sugar chains obtained from bovine colostrums k-casein. Biochim. Biophys. Acta 678:257-267.

Saito, T., and T. Itoh. 1992. Variations and distributions of O-glycosidically linked sugar chains in bovine k-casein. J. Dairy Sci. $75: 1768-1774$.

Sarker, S. A., T. H. Casswall, D. Mahalanabis, N. H. Alam, M. J. Albert, H. Brüssow, G. J. Fuchs, and L. Hammerström. 1998. Successful treatment of rotavirus diarrhea in children with immunoglobulin from immunized bovine colostrum. Pediatr. Infect. Dis. J. 17:1149-1154

Tateno, H., A. Mori, N. Uchiyama, R. Yabe, J. Iwaki, T. Shikanai, T. Angata, H. Narimatsu, and J. Hirabayashi. 2008. Glycoconjugate microarray based on an evanescent-field fluorescence-assisted detection principle for investigation of glycan-binding proteins. Glycobiology 18:789-798.

Uchida, K., N. Hiruta, H. Yamaguchi, K. Yamashita, K. Fujimura, and H. Yasui. 2012. Augmentation of cellular immunity and protection 
against influenza virus infection by bovine late colostrum in mice. Nutrition 28:442-446.

Wilson, N. L., L. J. Robinson, A. Donnet, L. Bovetto, N. H. Packer, and N. G. Karlsson. 2008. Glycoproteomics of milk: Difference in sugar epitopes on human and bovine milk fat globule membranes. J. Proteome Res. 7:3687-3696.

Yamada, K., S. Hyodo, M. Kinoshita, T. Hayakawa, and K. Kakehi. 2010. Hyphenated technique for releasing and MALDI MS analysis of O-glycans in mucin-type glycoprotein samples. Anal. Chem. 82:7436-7443.

Yamada, K., S. Hyodo, Y. K. Matsuno, M. Kinoshita, S. Z. Maruyama, Y. S. Osaka, E. Casal, Y. C. Lee, and K. Kakehi. 2007. Rapid and sensitive analysis of mucin-type glycans using an in-line flow glycan-releasing apparatus. Anal. Biochem. 371:52-61. 